\title{
A survey of wheelchair use by paraplegic individuals in Japan. Part 1: Characteristics of wheelchair cushions
}

\author{
Tadashi Sumiya $^{1}$, Kenji Kawamura ${ }^{2}$, Akihiro Tokuhiro ${ }^{3}$, Hideo Takechi $^{3}$ and Hajime Ogata ${ }^{4}$ \\ ${ }^{1}$ Rosai Rehabilitation Engineering Center, 1-10-5 Komei, Minato-ku, Nagoya, 455; ${ }^{2}$ Department of Physical Therapy, \\ School of Health Science, Kibi International University, 8 Igamachi, Takahashi, 716; ${ }^{3}$ Kibikogen Rehabilitation \\ Center, 7511 Nagasaka, Yoshikawa, Kayo-cho, Jobo-gun, 716-12; ${ }^{4}$ Department of Rehabilitation Medicine, University \\ of Occupational and Environmental Health, 1 Iseigaoka, Yahata Nishi-ku, Kita-kyushu, 807, Japan
}

\begin{abstract}
The characteristics of wheelchair cushions used by 218 paraplegic patients who lived independent lives were surveyed to clarify the present state of wheelchair cushioning for pressure sore prevention in Japan. Out of 586 cushions surveyed, 91.0\% were ready-made and the rest were custom-made. The outstanding popularity of polyurethane foam ready-made cushions $(76.3 \%)$ suggested that insufficient consideration was taken in the selection of cushions. Custom-made cushions displayed unique modifications to relieve contact pressure or to stabilize sitting posture, which should be systematically provided for all patients. The variety of cushion types and the frequent dissatisfaction with cushions seen in patients with current pressure sores suggested a strong demand for the effective prescription of cushions. Furthermore, $30 \%$ of all cushions had had an excessively prolonged use, indicating insufficient follow-up. A medical system including deliberate prescription and regular follow-up of wheelchair cushions should be established for the effective prevention of pressure sores.
\end{abstract}

Keywords: spinal cord injury; pressure sores; prevention; wheelchair cushion

\section{Introduction}

The pressure sore is a common complication of spinal cord injury and may cause physical, economical, and psycho-social problems. ${ }^{1}$ It may also result in many secondary complications and there may occasionally be a carcinomatous change. ${ }^{1}$ The sitting posture naturally creates high contact pressures at the ischial tuberosities, the coccyx, and in some cases the greater trochanters. The magnitude of contact pressure over the ischial tuberosities has been found to correlate well with incidence of the pressure sores. ${ }^{2}$ Therefore, wheelchairbound patients are always at the risk of pressure sores in these regions.

In recent years various designs of wheelchair cushions have been introduced to relieve high contact pressure at the bony prominences, ${ }^{3-9}$ and some have become commercially available in Japan. Although there are no universal prescription standards for these cushions, it is very important to select the appropriate cushion for patients to effectively prevent pressure sores. However, Japanese spinal cord injury patients commonly use ready-made wheelchair cushions of almost uniform shape and material, partly because they lack understanding of wheelchair cushions as medical therapeutic tools. To clarify the present state of wheelchair cushion use in Japanese paraplegic

Correspondence: T Sumiya patients, we performed direct interviews with over 200 who lived independent lives about their wheelchair cushions.

\section{Subjects and methods}

This survey was designed to obtain cross-sectional data from various regions of Japan in 1989.

The subjects surveyed included 218 self-supporting paraplegic patients, 201 males and 17 females (Table 1). All subjects became paraplegic as a result of a traumatic spinal cord injury. Although the neurological level of paralysis ranged from $\mathrm{T} 1$ to $\mathrm{L} 5$, the majority of patients $(159,72.9 \%)$ had a T10-L1 level. The survey was done in three different places; 145 subjects were surveyed in outpatient clinics of three major general hospitals (labor injury hospitals), 39 in industries, and 34 were interviewed at a wheelchair marathon. Ages ranged from 18 to 76 years (mean 43.3, SD 11.8), and body weight ranged from 30 to $93 \mathrm{~kg}$ (mean 57.3, SD 8.54). The period from onset of spinal cord injury varied between 1 and 59 years (mean 13.5, SD 8.95).

The subjects were interviewed directly about the development of pressure sores (previous and current), their physical condition, and their wheelchair cushions. The survey of pressure sores and physical condition are described in detail in Part 2 (Q.V). 
The wheelchair cushions were classified as readymade or custom-made. Custom-made cushions were made of ready-made material with modifications in shape or material. The type, shape, material, and thickness of cushions were recorded, and the subjects were questioned on the duration of cushion use and their feeling of satisfaction.

Table 1 Neurological level and paralysis of subjects surveyed

\begin{tabular}{lccc}
\hline \multirow{2}{*}{$\begin{array}{l}\text { Neurological } \\
\text { level }\end{array}$} & \multicolumn{3}{c}{ Paralysis } \\
Complete & Incomplete & Total \\
\hline T1 & 3 & 2 & 5 \\
T2 & 2 & 0 & 2 \\
T3 & 3 & 1 & 4 \\
T4 & 9 & 1 & 10 \\
T5 & 10 & 0 & 10 \\
T6 & 4 & 1 & 5 \\
T7 & 0 & 2 & 2 \\
T8 & 7 & 1 & 8 \\
T9 & 6 & 0 & 6 \\
T10 & 17 & 7 & 24 \\
T11 & 28 & 2 & 30 \\
T12 & 58 & 4 & 62 \\
L1 & 37 & 6 & 43 \\
L2 & 3 & 1 & 4 \\
L3 & 0 & 1 & 1 \\
L4 & 0 & 1 & 1 \\
L5 & 0 & 1 & 1 \\
Total & 187 & 31 & 218 \\
\hline
\end{tabular}

Furthermore, the influence of various cushions on the current pressure sore prevalence was statistically analyzed by using the $\chi^{2}$ test for independence $(P<0.05)$.

\section{Results}

From a total of 568 wheelchair cushions, $533(91.0 \%)$ were ready-made and the remainder $(53,9.0 \%)$ was custom-made (Table 2). Among the ready-made cushions, polyurethane foam was the most common material (76.3\% of all cushions), with some subjects choosing silicone gel flotation $(12.1 \%)$, and less frequently air-filled pads $(2.6 \%)$. Figure 1 shows characteristic configurations of these three ready-made wheelchair cushions. The polyurethane foam cushion had a medium density ${ }^{10}$ and a flat sitting surface. The silicone gel flotation consisted of a $5 \mathrm{~cm}$ thick silicone gel pad sandwiched between polyurethane foam pads. The air-filled pad was composed of a rubber envelope divided into several interconnected inflatable compartments, expanding to a thickness of $5 \mathrm{~cm}$ when filled.

The less common custom-made cushions exhibited unique characteristics in shape and material as shown in Figure 2. Some used harder polyurethane foam than normal or combined polyurethane foam with other kinds of material (eg cotton) to improve sitting stability, and some of the foam cushions had a cutout shape at the bony prominences to reduce high contact pressure, preventing skin break down.

There were 503 cushions of 179 patients without current pressure sores (sound-patients) and 83 cush-

Table 2 Wheelchair cushions surveyed

\begin{tabular}{|c|c|c|c|}
\hline \multirow[b]{2}{*}{ Classifications } & \multicolumn{3}{|c|}{ Number of wheelchair cushions } \\
\hline & Absent $(N a=503)$ & Present $(N p=83)$ & Total $(N=586)$ \\
\hline \multicolumn{4}{|l|}{ Type } \\
\hline (1) Ready-made cushions & $460(91.5)$ & $73(88.0)$ & $533(91.0)$ \\
\hline (a) Polyurethane foam & $391\left(77.7^{*}\right)$ & $56\left(67.5^{*}\right)$ & $447(76.3)$ \\
\hline (b) Silicone gel flotation & $60\left(11.9^{*}\right)$ & $11\left(13.3^{*}\right)$ & $71(12.1)$ \\
\hline (c) Air-filled pad & $9\left(1.8^{*}\right)$ & $6(7.2 *)$ & $15(2.6)$ \\
\hline (2) Custom-made cushions & $43\left(8.5^{*}\right)$ & $10\left(12.0^{*}\right)$ & $53(9.0)$ \\
\hline \multicolumn{4}{|l|}{ Thickness } \\
\hline$(1)<5 \mathrm{~cm}$ & $17(3.4)$ & $2(2.4)$ & $19(3.2)$ \\
\hline (2) $5-10 \mathrm{~cm}$ & $439(87.3)$ & $68(81.9)$ & $507(86.6)$ \\
\hline (3) $>10 \mathrm{~cm}$ & $47(9.3)$ & $13(15.7)$ & $60(10.2)$ \\
\hline \multicolumn{4}{|l|}{ Duration of use } \\
\hline (1) Short use $(<1$ year) & $140\left(27.8^{*}\right)$ & $16\left(19.3^{*}\right)$ & $156(26.6)$ \\
\hline (2) Long use & $363(72.2 *)$ & $67\left(80.7^{*}\right)$ & \\
\hline (a) $1-2$ years & $110(21.9)$ & $23(27.7)$ & $133(22.7)$ \\
\hline (b) $2-3$ years & $102(20.3)$ & $22(26.5)$ & $124(21.2)$ \\
\hline (c) 3-4 years & $71(14.1)$ & 7 (8.4) & $78(13.3)$ \\
\hline (d) $>4$ years & $80(15.9)$ & $15(18.1)$ & $95(16.2)$ \\
\hline \multicolumn{4}{|l|}{ Feeling of satisfaction } \\
\hline (1) satisfaction & $438(87.1)$ & $54(65.1)$ & $492(86.6)$ \\
\hline (2) dissatisfaction & $65(12.9)$ & $29(34.9)$ & $94(13.4)$ \\
\hline
\end{tabular}

( ) Percentages to each of $\mathrm{Na}, \mathrm{Np}$, and N. *Significant difference between absence and presence of current pressure sores at $P<0.05$ level 


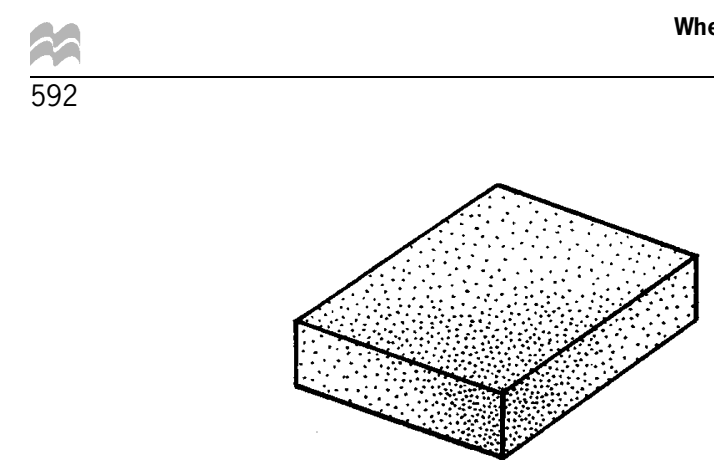

(a)

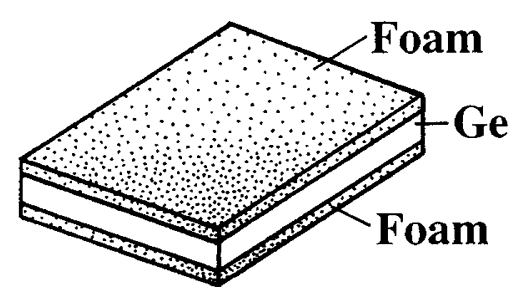

(b)

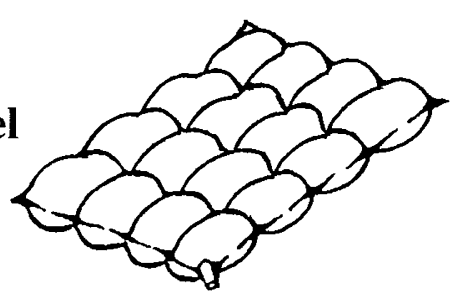

(c)

Figure 1 Ready-made cushions: (a) Polyurethane flat foam; (b) silicone gel flotation; and (c) air-filled pad

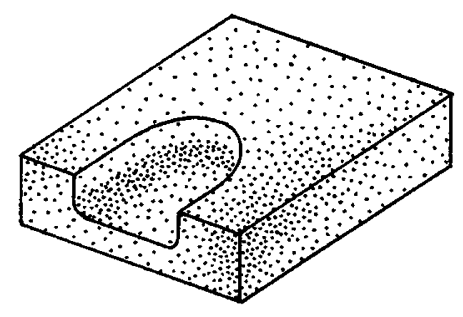

(a)

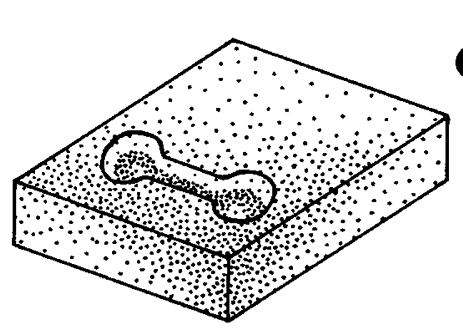

(b)

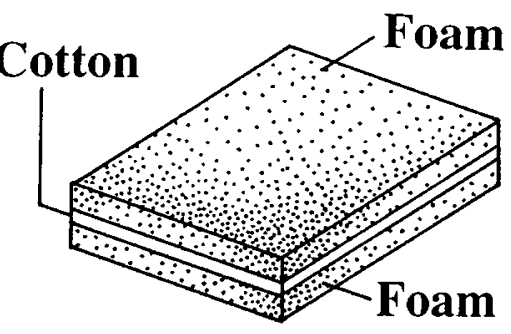

(c)

Figure 2 Three examples of custom-made cushions: (a) Cutout type-1; (b) cutout type-2; and (c) combined type

ions of 39 patients with current pressure sores (sorepatients), as shown in Table 2. Sore-patients used significantly larger variety of cushions than soundpatients, that is, the sore-patients' cushion group showed less frequent polyurethane foam cushions and more frequent silicone gel flotation, air-filled pads, and custom-made cushions than the soundpatients' cushion group.

The majority $(86.6 \%)$ of cushions had a thickness of $5-10 \mathrm{~cm}$, but some cushions were thicker than $10 \mathrm{~cm}$ $(10.2 \%)$ or thinner than $5 \mathrm{~cm}(3.2 \%)$.

Many patients posssessed more than one cushion and used them alternately, day by day, or case by case. The duration of use of each cushion varied from within 1 year to over 4 years. Most cushions were replaced after 1 to 3 years of use but some cushions (approximately 30\%) were in steady use for longer than 3 years. The proportion of cushions replaced after 1 year of duration in use (long use) was $80.7 \%$ in the sore-patients' cushion group; this was significantly higher than in the sound-patients' cushion group $(72.2 \%)$.

Most users $(175,80.3 \%)$ were satisfied with their cushions and the remainder $(43,19.7 \%)$ was dissatisfied. The total number of satisfied cushions was $492(86.6 \%)$ and that of dissatisfied cushions was $94(13.4 \%)$. The main reasons for dissatisfaction were poor durability and unfitted hardness. The proportion of dissatisfactory cushions was $34.9 \%$ in the sorepatients' cushion group; this was evidently higher than $12.9 \%$ in the sound-patients' cushion group.

\section{Discussion}

The high rate of males $(92.2 \%)$ in this survey sample largely exceeds the general ratio of male paraplegic patients in Japan $\left(81.2 \%\right.$ in 1990). ${ }^{11}$ This sample bias was attributed to the main survey setting being labor injury hospitals, because labor accidents more often involve males.

Each type of commercially available wheelchair cushion has functional characteristics that relate to the control of normal pressure, ${ }^{3-5,7-9}$ and tissue reaction to it, ${ }^{6}$ shearing force, ${ }^{11}$ humidity, ${ }^{12}$ temperature, ${ }^{8,12}$ and sitting stability, ${ }^{10}$ and will also have a variable weight, ${ }^{4,6}$ cost, ${ }^{6}$ and durability. ${ }^{1}$ Effective pressure sore prevention requires careful consideration of these facts and techniques.

The effectiveness of a clinic specifically for the prevention of pressures sores has been reported elsewhere in the world, ${ }^{2,13,14}$ but has not been reported in Japan. In such a clinic, patients acquire basic knowledge and proper technique for pressure relief, and interface pressure is measured routinely to detect the risk of pressure sores and to evaluate the fit of cushions. The cutout foam type of cushion has been reported to be the most effective cushion for redistribution of contact pressure under the ischial tuberosities. $^{2}$

Many of the custom-made cushions surveyed had specific modifications (Figure 2); the cutout type was designed on the same basis as the cutout cushions described above, and the combined type was created to 
obtain better sitting stability on the same basis as a modular cushion system. ${ }^{8}$ We believe these modifications should be carried out systematically, as a medical prescription for all patients who need effective pressure relief or sitting stability. Previous studies have revealed that no single cushion was clearly ideal from all aspects, and therefore the selection and fitting of cushions to patients should be done on an individual basis. $^{7,9}$

The outstanding popularity of the polyurethane foam ready-made cushion and the high satisfaction with the use of this kind of cushion suggest this type of cushion is superior to others. Polyurethane foam has many advantages; it is readily available, there is a large variety of types, it is inexpensive, lightweight, easily transferred, and breathable. These various advantages make this cushion universal and very popular, benefiting large numbers of Japanese paraplegic patients. However, the significant proportion of patients with current pressure sores $(39,17.9 \%)$ and of patients dissatisfied with cushions $(43,19.7 \%)$ need to be reduced by more careful consideration, given to the selection and modification of wheelchair cushions for each individual patient. The almost uniform application of polyurethane foam ready-made cushions to the subjects of this survey should be reconsidered.

The frequent dissatisfaction of the sore-patients with ready-made polyurethane foam cushions made the patients search for various cushions designed for effective prevention of their pressure sores. As a result, the sore-patients possessed a significantly larger variety of cushions than the sound-patients.

The lifetime of cutout foam cushions is limited to within 6-12 months because they may only be used in one direction, and therefore receive repeated stress on the same area. ${ }^{2,15}$ In contrast, the lifetime of flat foam cushions varies, partly because they may be used in multiple directions or sides so that stress is dispersed across the cushion and partly because many of them are used alternately. However, there is no foundation for a useful duration of flat foam cushions of longer than 1 year. Moreover, foam cushions deteriorate with time even without use, and contact pressure may reach unacceptable levels after a long period. ${ }^{1}$ Therefore, the excessively prolonged use of foam cushions is generally undesirable. The significantly higher proportion of long use in the sore-patients' cushion group than in the sound-patients' cushion group suggests that the use of deteriorated cushion material has probably played an important role in the persistence of pressure sores. This resulted from an insufficient follow-up of prescribed cushions. Suitable follow-up systems are required to encourage the patients to use their cushions correctly.

Although various functional tests for ready-made wheelchair cushions, including pressure evaluation, have been reported in Japan since this survey, neither improvement of prescription nor systematization of follow-up has been reported as yet. A medical system including deliberate prescription and regular follow-up of wheelchair cushions should be established to individually fit the most appropriate cushions, not only ready-made but custom-made, for paraplegic patients for the effective prevention of pressure sores in Japan.

\section{Conclusion}

A survey of 218 Japanese paraplegic patients who live independent lives revealed insufficient application of wheelchair cushions for pressure sore prevention. Three major findings support this conclusion: (1) There was a markedly high prevalence of polyurethane foam cushions $(76.3 \%)$ without modification; (2) effective modifications were only seen in a minority of cushions that were custom-made $(9.0 \%)$; and (3) the majority of cushions $(73.4 \%)$ were in prolonged use (over 1 year). Furthermore, the high variety of cushions used and the high frequency of dissatisfaction with cushions in patients with current pressure sores suggested a strong demand for prescription and fit of appropriate wheelchair cushions. A medical system including deliberate prescription and regular follow-up of wheelchair cushions should be established for the prevention of pressure sores in patients with paraplegia in Japan.

\section{Acknowledgements}

These surveys, for the two papers were supported by the Japanese Labor Welfare Corporation. The authors would like to acknowledge the role of Dr Akio Nakajima, Dr Torakichi Inoue, Dr Kimio Shimada, and Dr Osamu Yoshimura, who contributed greatly to the present data collection of this survey.

\section{References}

1 Yarkony GM. Pressure ulcers: a review. Arch Phys Med Rehabil 1994; 75: $908-917$.

2 Mooney V, Einbund MJ, Rogers JE, Stauffer ES. Comparison of pressure distribution qualities in seat cushions. Bull Prosthet Res 1971; BPR 10-15: 129-143.

3 Cochran GVB, Slater G. Experimental evaluation of wheelchair cushions: report of a pilot study. Bull Prosthet Res 1973; BPR 10-20: $29-61$.

4 Souther SG, Carr SD, Vistnes LM. Wheelchair cushions to reduce pressure under bony prominences. Arch Phys Med Rehabil 1974; 55: $460-464$

5 DeLateur BJ, Berni R, Hongladarom T, Giaconi R. Wheelchair cushions designed to prevent pressure sores: an evaluation. Arch Phys Med Rehabil 1976; 57: 129-135.

6 Seymour RJ, Lacefield WE. Wheelchair cushion effect on pressure and skin temperature. Arch Phys Med Rehabil 1985; 66: $103-108$.

7 Ferguson-Pell MW. Seat cushion selection. J Rehabil Res Dev 1990; Suppl 2: $49-73$.

8 Bar CA. Evaluation of cushions using dynamic pressure measurement. Prosthet Orthot Int 1991; 15: $232-240$.

9 McFadyen GM, Stoner DL. Polyurethane foam wheelchair cushions, retention of supportive properties. Arch Phys Med Rehabil 1980; 61: $234-237$.

10 Hobson DA. Comparative effects of posture on pressure and shear at the body-seat interface. J Rehabil Res Dev 1992; 29: $21-$ 31 . 
11 Shingu H, Ikata T, Katoh S, Akatsu T. Spinal cord injuries in Japan, a nationwide epidemiological survey in 1990. Paraplegia 1994; 32: $3-8$.

12 Stewart SFC, Palmieri V, Cochran GVB. Wheelchair cushion effect on skin temperature, heat flux, and relative humidity. Arch Phys Med Rehabil 1980; 61: 229-233.

13 Krouskop TA, Noble PC, Garber SL, Spencer WA. The effectiveness of preventive management in reducing the occurrence of pressure sores. J Rehabil Res Dev 1983; 20: 74-83.

14 Dover H, Pickard W, Swain I, Grundy D. The effectiveness of a pressure clinic in preventing pressure sores. Paraplegia 1992; 30: $267-272$.

15 Ferguson-Pell MW, Wilkie IC, Reswick JB, Barbenel JC. Pressure sore prevention for the wheelchair-bound spinal injury patient. Paraplegia 1980; 18: $42-51$. 